\title{
Isospin-dependent pion in-medium effects on the charged-pion ratio in heavy ion collisions
}

\author{
Jun $\mathrm{Xu},{ }^{1, *}$ Che Ming Ko, ${ }^{2, \dagger}$ and Yongseok $\mathrm{Oh}^{3, \dagger, \S}$ \\ ${ }^{1}$ Cyclotron Institute, Texas A\&M University, College Station, Texas 77843, USA \\ ${ }^{2}$ Cyclotron Institute and Department of Physics and Astronomy, Texas A\&M University, College Station, Texas 77843, USA \\ ${ }^{3}$ Korea Institute of Science and Technology Information, Daejeon 305-806, Korea
}

(Received 9 June 2009; revised manuscript received 8 January 2010; published 25 February 2010)

\begin{abstract}
Using results from the chiral perturbation theory for the $s$-wave interaction and the $\Delta$-resonance model for the $p$-wave interaction of pions with nucleons, we evaluated the spectral functions of pions in asymmetric nuclear matter with unequal proton and neutron densities. We find that in hot dense neutron-rich matter the strength of the spectral function of positively charged pions at low energies is somewhat larger than that of negatively charged pions. In a thermal model, this isospin-dependent effect slightly reduces the ratio of negatively charged to positively charged pions that are produced in heavy ion collisions induced by radioactive beams. The relevance of our results to the determination of the nuclear symmetry energy from the measured ratio of negatively to positively charged pions produced in heavy ion collisions is discussed.
\end{abstract}

DOI: 10.1103/PhysRevC.81.024910

PACS number(s): 21.65.Jk, 21.65.Ef, 25.75.Dw

\section{INTRODUCTION}

The nuclear symmetry energy is the energy needed per nucleon to convert all protons in a symmetric nuclear matter to neutrons. Knowledge on the density dependence of the nuclear symmetry energy is important for understanding the dynamics of heavy ion collisions induced by radioactive beams, the structure of exotic nuclei with large neutron or proton excess, and many important issues in nuclear astrophysics [1-4]. At normal nuclear matter density, the nuclear symmetry energy has long been known to have a value of about $30 \mathrm{MeV}$ from fitting the binding energies of atomic nuclei with the liquid-drop mass formula. Somewhat stringent constraints on the nuclear symmetry energy below the normal nuclear density were also obtained during the past few years from studies of the isospin diffusion [5-8] and isoscaling [9] in heavy-ion reactions, the size of neutron skin in heavy nuclei [10], and the isotope dependence of the giant monopole resonances in even-A Sn isotopes [11]. For nuclear symmetry energy at high densities, transport model studies showed that the ratio of negatively to positively charged pions produced in heavy ion collisions with neutron-rich nuclei is sensitive to its stiffness [12-14] and there was an attempt [15] to extract the symmetry energy at suprasaturation densities from the FOPI data on the $\pi^{-} / \pi^{+}$ratio in central heavy ion collisions at SIS/GSI [16].

The transport model used in Refs. [12,13,15] neglects, however, medium effects on pions, although it includes those on nucleons and produced $\Delta$ resonances through their isospin-dependent mean-field potentials and scattering cross sections. It is well known that pions interact strongly in a nuclear medium as a result of their $p$-wave interactions

\footnotetext{
*xujun@ comp.tamu.edu

†ko@comp.tamu.edu

${ }^{\ddagger}$ yoh@kisti.re.kr

${ }^{\S}$ Address after March 1, 2010: School of Physics and Energy Sciences, Kyungpook National University, Daegu 702-701, Korea.
}

through couplings to the nucleon-particle-nucleon-hole and $\Delta$-particle-nucleon-hole ( $\Delta$-hole) excitations, leading to the softening of their dispersion relations or increased strength of their spectral functions at low energies [17-22]. Furthermore, it was shown in Ref. [23] that, in asymmetric nuclear matter with different proton and neutron fractions, these medium effects depend on the charge of the pion. Including the $p$-wave, pion medium effects in the transport model was previously shown to enhance the production of low-energy pions in high-energy heavy ion collisions, although it does not affect the total pion yield $[24,25]$. Properties of pions in the nuclear medium are also modified by their $s$-wave interactions with nucleons. Studies based on chiral perturbation theory showed that, in asymmetric nuclear matter, this effect also depends on the charge of the pion [26], leading to different in-medium masses for negatively and positively charged pions. Since pions of different charges are modified differently in asymmetric nuclear matter, it is of interest to study how such isospindependent medium effects will affect the ratio of negatively to positively charged pions produced in heavy ion collisions.

This article is organized as follows. We first evaluate in Sec. II the spectral functions of pions and delta-resonances in hot dense asymmetric nuclear matter. Using these spectral functions, we then study in Sec. III the charged pion ratio in a thermal model and compare the results with the available experimental data. We conclude with a summary in Sec. IV.

\section{MEDIUM EFFECTS}

\section{A. Pion in-medium properties}

Pions in nuclear medium acquire self-energies through their $s$-wave and $p$-wave interactions. In asymmetric nuclear matter, the pion self-energy further depends on the charge of the pion.

\section{Pion-nucleon s-wave interaction}

The contribution of the pion-nucleon $s$-wave interaction to the pion self-energy was calculated in Ref. [26] up to the 
two-loop order in chiral perturbation theory. In asymmetric nuclear matter of proton density $\rho_{p}$ and neutron density $\rho_{n}$, the resulting pion self-energy depends on the charge of the pion and for $\pi^{-}, \pi^{+}$, and $\pi^{0}$, their self-energies due to the $s$-wave interactions are given, respectively, by

$$
\begin{aligned}
\Pi_{s}^{-}\left(\rho_{p}, \rho_{n}\right)= & \rho_{n}\left[T_{\pi N}^{-}-T_{\pi N}^{+}\right]-\rho_{p}\left[T_{\pi N}^{-}+T_{\pi N}^{+}\right] \\
& +\Pi_{\text {rel }}^{-}\left(\rho_{p}, \rho_{n}\right)+\Pi_{\text {cor }}^{-}\left(\rho_{p}, \rho_{n}\right), \\
\Pi_{s}^{+}\left(\rho_{p}, \rho_{n}\right)= & \Pi_{s}^{-}\left(\rho_{n}, \rho_{p}\right), \\
\Pi_{s}^{0}\left(\rho_{p}, \rho_{n}\right)= & -\left(\rho_{p}+\rho_{n}\right) T_{\pi N}^{+}+\Pi_{\text {cor }}^{0}\left(\rho_{p}, \rho_{n}\right) .
\end{aligned}
$$

Here, $T^{ \pm}$are the isospin-even and isospin-odd $\pi N s$-wave scattering $T$-matrices, which are given by the one-loop contribution in chiral perturbation theory and have the empirical values $T_{\pi \mathrm{N}}^{-} \approx 1.847$ and $T_{\pi \mathrm{N}}^{+} \approx-0.045 \mathrm{fm}$ extracted from the energy shift and width of the 1 s level in the pionic $\mathrm{H}$ atom. The term $\Pi_{\text {rel }}^{-}$is due to the relativistic correction, whereas the terms $\Pi_{\text {cor }}^{-}$and $\Pi_{\text {cor }}^{0}$ are the contributions from the two-loop order in chiral perturbation theory. Their expressions can be found in Ref. [26], where it is also shown that in nuclear matter of density $\rho=0.165 \mathrm{fm}^{-3}$ and isospin asymmetry $\delta=\left(\rho_{n}-\rho_{p}\right) /\left(\rho_{n}+\rho_{p}\right)=0.2$, such as in the interior of a $\mathrm{Pb}$ nucleus, changes of the pion masses due to their $s$-wave interactions are $\Delta m_{\pi^{-}}=13.8, \Delta m_{\pi^{+}}=-1.2$, and $\Delta m_{\pi^{0}}=$ 6.1 MeV. Compared to their masses in free space, the $\pi^{-}$ mass increases whereas the $\pi^{+}$mass decreases in neutron-rich nuclear matter.

\section{Pion-nucleon p-wave interaction}

For the $p$-wave interactions of pions in nuclear matter, we consider only the dominant $\Delta$-hole excitations as in Ref. [27], as the contribution from the nucleon particle-hole excitations is known to be small. For a pion of isospin state $m_{t}$, energy $\omega$, and momentum $k$ in a hot nuclear medium at temperature $T$, its self-energy due to the $p$-wave interaction is then given by

$$
\begin{aligned}
\Pi_{0}^{m_{t}} \approx & \frac{4}{3}\left(\frac{f_{\Delta}}{m_{\pi}}\right)^{2} k^{2} F_{\pi}^{2}(k) \sum_{m_{\tau}, m_{T}}\left|\left\langle\frac{3}{2} m_{T} \mid 1 m_{t} \frac{1}{2} m_{\tau}\right\rangle\right|^{2} \\
& \times \int \frac{d^{3} p}{(2 \pi)^{3}} \frac{1}{e^{\left(m_{N}+p^{2} / 2 m_{N}+U_{N}^{m_{\tau}}-\mu_{B}-2 m_{\tau} \mu_{Q}\right) / T}+1} \\
& \times\left(\frac{1}{\omega-\omega_{m_{T}}^{+}}+\frac{1}{-\omega-\omega_{m_{T}}^{-}}\right)
\end{aligned}
$$

with

$$
\begin{aligned}
\omega_{m_{T}}^{ \pm} \approx & m_{\Delta}+U_{\Delta}^{m_{T}}+\frac{(\vec{k} \pm \vec{p})^{2}}{2 m_{\Delta}}-i \frac{\Gamma_{\Delta}^{m_{T}}}{2} \\
& -m_{N}-U_{N}^{m_{\tau}}-\frac{p^{2}}{2 m_{N}} .
\end{aligned}
$$

In the above equation, $m_{\Delta} \simeq 1232 \mathrm{MeV}$ is the mass of $\Delta$ resonance; $f_{\Delta} \simeq 3.5$ is the $\pi N \Delta$ coupling constant, and $F_{\pi}(k)=\left[1+0.6\left(k^{2} / m_{\pi}^{2}\right)\right]^{-1 / 2}[28]$ is the $\pi N \Delta$ form factor determined by fitting the decay width $\simeq 118 \mathrm{MeV}$ of $\Delta$ resonance in free space. The summation in Eq. (2) is over the nucleon isospin state $m_{\tau}$ and the $\Delta$ resonance isospin state
$m_{T}$; and the factor $\left\langle\frac{3}{2} m_{T} \mid 1 m_{t} \frac{1}{2} m_{\tau}\right\rangle$ is the Clebsch-Gordan coefficient from the isospin coupling of a pion with nucleon and $\Delta$ resonance. The momentum integration is over that of nucleons in the nuclear matter given by a Fermi-Dirac distribution with $\mu_{B}$ and $\mu_{Q}$ being, respectively, the baryon and charge chemical potentials determined by charge and baryon number conservations; $U_{N}^{m_{\tau}}$ is the mean-field potential of nucleons of isospin state $m_{\tau}$ in nuclear matter; and $\Gamma_{\Delta}^{m_{T}}$ and $U_{\Delta}^{m_{T}}$ are, respectively, the width and mean-field potential of $\Delta$ resonance of isospin state $m_{T}$.

For the nucleon mean-field potential $U_{N}^{m_{\tau}}$, we use that from the momentum-independent (MID) interaction [1]

$$
U_{N}^{m_{\tau}}\left(\rho_{B}, \delta_{\text {like }}\right)=\alpha\left(\frac{\rho_{B}}{\rho_{0}}\right)+\beta\left(\frac{\rho_{B}}{\rho_{0}}\right)^{\gamma}+U_{\text {asy }}^{m_{\tau}}\left(\rho_{B}, \delta_{\text {like }}\right),
$$

with

$$
\begin{aligned}
& U_{\text {asy }}^{m_{\tau}}\left(\rho_{B}, \delta_{\text {like }}\right) \\
& =-4\left\{F(x)\left(\frac{\rho_{B}}{\rho_{0}}\right)+[18.6-F(x)]\left(\frac{\rho_{B}}{\rho_{0}}\right)^{G(x)}\right\} m_{\tau} \delta_{\text {like }} \\
& \quad+[18.6-F(x)][G(x)-1]\left(\frac{\rho_{B}}{\rho_{0}}\right)^{G(x)} \delta_{\text {like }}{ }^{2}
\end{aligned}
$$

being the nucleon symmetry potential. The parameters $\alpha=$ $-293.4 \mathrm{MeV}, \beta=240.1 \mathrm{MeV}$, and $\gamma=1.216$ are chosen to give a compressibility of $212 \mathrm{MeV}$ and a binding energy per nucleon of $-16 \mathrm{MeV}$ for symmetric nuclear matter at saturation or normal nuclear density $\rho_{0}=0.16 \mathrm{fm}^{-3}$. The nucleon symmetry potential $U_{\text {asy }}^{m_{\tau}}\left(\rho_{B}, \delta_{\text {like }}\right)$ depends on the baryon density $\rho_{B}=\rho_{n}+\rho_{p}+\rho_{\Delta^{-}}+\rho_{\Delta^{0}}+\rho_{\Delta^{+}}+\rho_{\Delta^{++}}$ and the isospin asymmetry $\delta_{\text {like }}=\left(\rho_{n}-\rho_{p}+\rho_{\Delta^{-}}-\rho_{\Delta^{++}}+\right.$ $\left.\rho_{\Delta^{0}} / 3-\rho_{\Delta^{+}} / 3\right) / \rho_{B}$ of asymmetric hadronic matter, which is a generalization of the isospin asymmetry $\delta$ usually defined for asymmetric nuclear matter without $\Delta$ resonances [12]. The nucleon mean-field potential also depends on the stiffness of nuclear symmetry energy through the parameter $x$ via the functions $F(x)$ and $G(x)$. We consider the three cases of $x=0, x=0.5$, and $x=1$ with corresponding values $F(x=0)=129.98$ and $G(x=0)=1.059, F(x=0.5)=$ 85.54 and $G(x=0.5)=1.212$, and $F(x=1)=107.23$ and $G(x=1)=1.246$. The resulting nuclear symmetry energy becomes increasingly softer as the value of $x$ increases, with $x=1$ giving a nuclear symmetry energy that becomes negative at about three times the normal nuclear matter density as shown in Fig. 1. These symmetry energies reflect the uncertainties in the theoretical predictions on the stiffness of nuclear symmetry energy at high densities.

For the mean-field potentials of $\Delta$ resonances, their isoscalar potentials are assumed to be the same as those of nucleons and their symmetry potentials are taken to be the average of those for neutrons and protons with the weighting factors depending on the charge state of $\Delta$ resonances [28] $U_{\text {asy }}^{\Delta^{++}}=U_{\text {asy }}^{p}, U_{\text {asy }}^{\Delta^{+}}=\frac{2}{3} U_{\text {asy }}^{p}+\frac{1}{3} U_{\text {asy }}^{n}, U_{\text {asy }}^{\Delta^{0}}=\frac{1}{3} U_{\text {asy }}^{p}+\frac{2}{3} U_{\text {asy }}^{n}$, and $U_{\text {asy }}^{\Delta^{-}}=U_{\text {asy }}^{n}$.

Including the short-range $\Delta$-hole repulsive interaction via the Migdal parameter $g^{\prime}$, which has values $1 / 3 \leqslant g^{\prime} \leqslant 0.6$ 


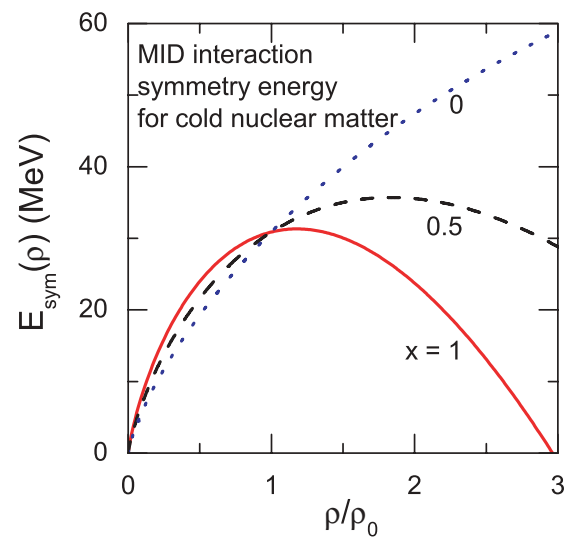

FIG. 1. (Color online) Nuclear symmetry energies as functions of nuclear density from the MID interaction for different values of the symmetry energy parameter $x$.

[17-22], modifies the pion self-energy due to the $p$-wave interaction to

$$
\Pi_{p}^{m_{t}}=\frac{\Pi_{0}^{m_{t}}}{1-g^{\prime} \Pi_{0}^{m_{t}} / k^{2}}
$$

\section{Pion spectral function}

In terms of the pion self-energies $\Pi_{s}^{m_{\tau}}$ and $\Pi_{p}^{m_{\tau}}$ due to the pion-nucleon $s$-wave and $p$-wave interactions in nuclear medium, the pion in-medium propagator is given by

$$
D^{m_{t}}(\omega, k)=\frac{1}{\omega^{2}-k^{2}-m_{\pi}^{2}-\Pi_{s}^{m_{t}}-\Pi_{p}^{m_{t}}(\omega, k)} .
$$

The pion spectral function $S_{\pi}^{m_{t}}(\omega, k)$ is related to the imaginary part of the pion in-medium propagator through

$$
S_{\pi}^{m_{t}}(\omega, k)=-\frac{1}{\pi} \operatorname{Im} D^{m_{t}}(\omega, k) .
$$

\section{B. Delta resonance in-medium properties}

The modification of the pion properties in the nuclear medium affects the decay width and mass distribution of $\Delta$ resonances. For a $\Delta$ resonance of isospin state $m_{T}$ and mass $M$ and at rest in nuclear matter, its decay width is given by [27]

$$
\begin{aligned}
\Gamma_{\Delta}^{m_{T}}(M) & \\
\approx & -2 \sum_{m_{\tau}, m_{t}}\left|\left\langle\frac{3}{2} m_{T} \mid 1 m_{t} \frac{1}{2} m_{\tau}\right\rangle\right|^{2} \\
& \times \int \frac{d^{3} \mathbf{k}}{(2 \pi)^{3}}\left(\frac{f_{\Delta}}{m_{\pi}}\right)^{2} F_{\pi}^{2}(k)\left[\frac{1}{z_{\pi}^{-1} e^{\left(\omega-m_{t} \mu_{Q}\right) / T}-1}+1\right] \\
& \times\left[1-\frac{1}{e^{\left(m_{N}+k^{2} / 2 m_{N}+U_{N}^{m_{\tau}}-\mu_{B}-2 m_{\tau} \mu_{Q}\right) / T}+1}\right] \\
& \times \operatorname{Im}\left\{\frac{k^{2}}{3} \frac{D^{m_{t}}(\omega, k)}{\left[1-g^{\prime} \Pi_{0}^{m_{t}}(\omega, k) / k^{2}\right]^{2}}+g^{\prime 2} \frac{\Pi_{p}^{m_{t}}(\omega, k)}{k^{2}}\right\} .
\end{aligned}
$$

In the above equation, the first term in the last line is due to the decay of the $\Delta$ resonance to the pion, but corrected by the contact interaction at the $\pi N \Delta$ vertex, while the second term contains the contribution from its decay to the $\Delta$-hole state without coupling to the pion. The two temperature-dependent factors in the momentum integral take into account, respectively, the Bose enhancement for the pion and the Pauli blocking of the nucleon. To include a possible chemical nonequilibrium effect, a fugacity parameter $z_{\pi}$ is introduced for pions. The pion energy $\omega$ is determined from energy conservation $M+U_{\Delta}^{m_{T}}=\omega+m_{N}+k^{2} / 2 m_{N}+U_{N}^{m_{\tau}}$. The resulting mass distribution of $\Delta$ resonances is then given by

$$
P_{\Delta}(M)=A \frac{\Gamma_{\Delta}^{m_{T}}(M) / 2}{\left(M-m_{\Delta}\right)^{2}+\Gamma_{\Delta}^{m_{T}{ }^{2}}(M) / 4},
$$

where $A$ is a normalization constant to ensure the integration of $P_{\Delta}(M)$ over $M$ is 1 .

\section{Results}

Equations (2), (6), and (8) are solved self-consistently to obtain the pion spectral functions and the mass distributions of $\Delta$ resonances in asymmetric nuclear matter. The results obtained with the Migdal parameter $g^{\prime}=1 / 3$ are illustrated in Figs. 2 and 3 for an asymmetric nuclear matter of isospin asymmetry $\delta_{\text {like }} \simeq 0.135$, twice the normal nuclear matter density $\rho_{B}=2 \rho_{0}$, temperature $T \simeq 43.6 \mathrm{MeV}$, and chemical potentials $\mu_{B} \simeq 942$ and $\mu_{Q} \simeq-18.5 \mathrm{MeV}$, corresponding to those to be used in the thermal model and also similar to those reached in the isospin-dependent Boltzmann-UehlingUhlenbeck (IBUU) transport model with the nuclear symmetry energy $x=1$ for central $\mathrm{Au}+\mathrm{Au}$ collisions at the beam energy of $0.4 A \mathrm{GeV}$ [15]. Shown in Fig. 2 are the pion spectral functions as functions of pion energy for different values of the pion momentum. It is seen that for low pion momenta the spectral function at low energies has a larger

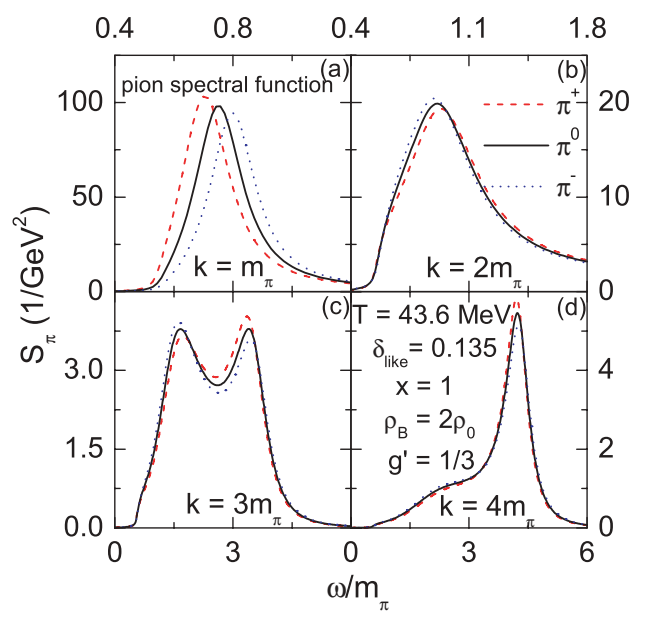

FIG. 2. (Color online) Spectral functions of pions in asymmetric nuclear matter of density $2 \rho_{0}$ and isospin asymmetry $\delta_{\text {like }}=0.135$ as functions of pion energy for different pion momenta of (a) $m_{\pi}$, (b) $2 m_{\pi}$, (c) $3 m_{\pi}$, and (d) $4 m_{\pi}$. All are calculated with the Migdal parameter $g^{\prime}=1 / 3$. 


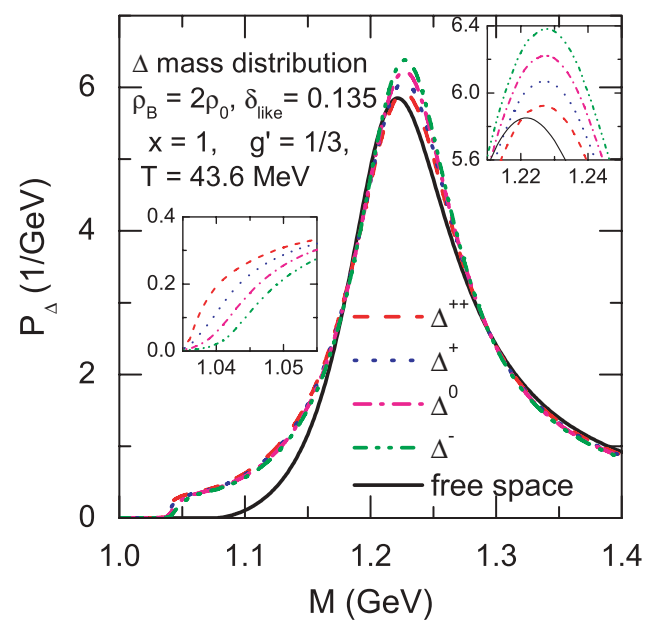

FIG. 3. (Color online) Mass distributions of $\Delta$ resonances at rest in asymmetric nuclear matter of density $2 \rho_{0}$ and isospin asymmetry $\delta_{\text {like }}=0.135$. The solid line corresponds to that in free space. The distributions near the threshold and at the peak are enlarged in the insets.

strength for $\pi^{+}$(dashed line) than for $\pi^{0}$ (solid line), which has a strength larger than that for $\pi^{-}$(dotted line) and this behavior is reversed at high pion energies. Figure 3 shows the mass distributions of $\Delta$ resonances at rest in asymmetric nuclear matter as functions of mass. One sees that it is similar to that in free space (solid line) as a result of the cancellation between the pion in-medium effects, which enhance the strength at low masses and the Pauli-blocking of the nucleon from delta decay, which reduces the strength at low masses. This is consistent with the observed similar energy dependence of the photo-proton and photo-nucleus absorption cross sections around the $\Delta$ resonance [29]. Furthermore, whereas the strength around the peak of the $\Delta$ resonance mass distribution decreases with increasing charge of $\Delta$ resonance due to nonzero isospin asymmetry of the nuclear medium that near the threshold increases with increasing $\Delta$ resonance charge. We note that the pion-nucleon $s$-wave and $p$-wave interactions have opposite effects on the in-medium properties of pions and delta resonances. While the pion-nucleon $s$-wave interaction increases the $\pi^{-}$mass and reduces the $\pi^{+}$mass, the pion-nucleon $p$-wave interaction softens the dispersion relation of $\pi^{-}$more than that of $\pi^{+}$. As a result, including only medium effects due to the pion-nucleon $p$-wave interaction will lead to an opposite result (i.e., for low momentum pions the spectral function at low energies has a larger strength for $\pi^{-}$than for $\pi^{+}$) although the strength around the peak of the $\Delta$ resonance mass distribution still decreases with increasing charge of $\Delta$ resonance.

\section{CHARGED PION RATIO IN HOT DENSE ASYMMETRIC NUCLEAR MATTER}

To see the isospin-dependent pion in-medium effects on the $\pi^{-} / \pi^{+}$ratio in heavy ion collisions, we use a thermal model, which assumes that pions are in thermal equilibrium with nucleons and $\Delta$ resonances [30]. The density of particle species $i$ is then given by

$$
\rho_{i} \approx g_{i} \int \frac{d^{3} \mathbf{k}}{(2 \pi)^{3}} d \omega^{n_{i}} S_{i}(\omega, k) \frac{1}{z_{i}^{-1} e^{\left(\omega-B_{i} \mu_{B}-Q_{i} \mu_{Q}\right) / T} \pm 1} .
$$

In the above equation, $g_{i}, B_{i}, Q_{i}$, and $z_{i}$ are the degeneracy, baryon number, charge, and fugacity of the particle. The exponent $n_{i}$ is 2 for pions and 1 for nucleons and $\Delta$ resonances. For pions, we use the spectral functions $S_{\pi}^{m_{t}}(\omega, k)$ calculated previously. For the spectral functions of $\Delta$ resonances, we neglect their momentum dependence and thus replace the integration over energy $\omega$ by that over mass $M$. The $\omega$ in the Fermi-Dirac distribution for $\Delta$ resonances is then simply $\omega=M+k^{2} / 2 M+U_{\Delta}^{m_{T}}$. For nucleons, their spectral functions are taken to be delta functions if we neglect the imaginary part of their self-energies $S_{N}^{m_{\tau}}(\omega, k)=\delta\left(\omega-m_{N}-\right.$ $\left.k^{2} / 2 m_{N}-U_{N}^{m_{\tau}}\right)$.

According to studies based on transport models [12,15,24], the total number of pions and $\Delta$ resonances in heavy ion collisions reaches a maximum value when the colliding matter achieves the maximum density and remains essentially constant during the expansion of the matter. For $\mathrm{Au}+$ $\mathrm{Au}$ collisions at the beam energy of $0.4 \mathrm{~A} \mathrm{GeV}$, for which the $\pi^{-} / \pi^{+}$ratio was measured by the FOPI Collaboration at GSI [16], the IBUU transport model gives a maximum density that is about twice the normal nuclear matter density and is insensitive to the stiffness of the nuclear symmetry energy, as it is mainly determined by the isoscalar part of the nuclear equation of state [15]. We thus use this density in the thermal model. The temperature in the thermal model is determined by fitting the measured pion to nucleon ratio, which is about 0.014 including pions and nucleons from decays of $\Delta$ resonances [16], without medium effects and with unity fugacity parameters for all particles and the value is $T \simeq$ $43.6 \mathrm{MeV}$. The assumption that pions and $\Delta$ resonances are in chemical equilibrium is consistent with the short chemical equilibration times estimated from the pion and $\Delta$ resonance production rates. The isospin asymmetry of the hadronic matter is then taken to be $\delta_{\text {like }} \simeq 0.080,0.106$, and 0.143 , corresponding to net charge densities of $0.920 \rho_{0}, 0.894 \rho_{0}$, and $0.857 \rho_{0}$, for the three symmetry energies given by $x=0$, 0.5 , and 1 , respectively, to reproduce the $\pi^{-} / \pi^{+}$ratios of $2.20,2.40$, and 2.60 predicted by the IBUU transport model of Ref. [15] using corresponding symmetry energies without pion in-medium effects. Since medium effects enhance the pion and $\Delta$ resonance densities, to maintain same pion to nucleon ratio as the measured one requires fugacity parameters for pions and $\Delta$ resonances to be less than 1 . Also, the pion in-medium effects were shown to affect only slightly the pion and the $\Delta$ resonance abundance [24], indicating that both pions and $\Delta$ resonances are out of chemical equilibrium with nucleons when medium effects are included, as expected from estimated increasing pion and $\Delta$ resonance chemical equilibration times as a result of medium effects. Because of the small number of pions (about $0.3 \%$ ) and $\Delta$ (about $1.1 \%$ ) resonances in the matter, the density, temperature, and net charge density of the hadronic matter are expected to remain unchanged when pion in-medium effects are introduced. They lead to, however, 


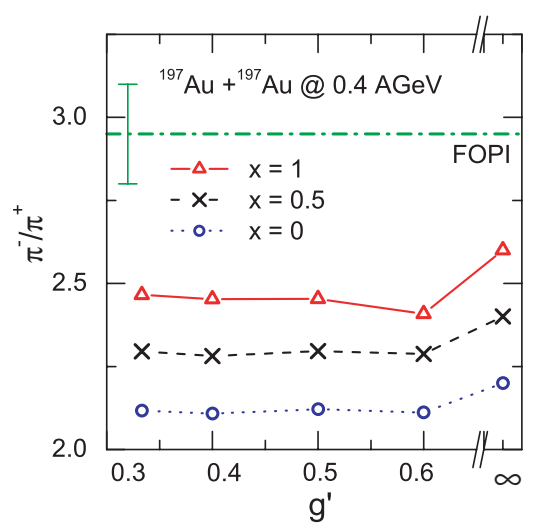

FIG. 4. (Color online) The $\pi^{-} / \pi^{+}$ratio in $\mathrm{Au}+\mathrm{Au}$ collisions at the beam energy of $0.4 \mathrm{~A} \mathrm{GeV}$ for different values of nuclear symmetry energy parameter $(x=0,0.5$, and 1$)$ and the Migdal parameter $g^{\prime}=1 / 3,0.4,0.5$, and 0.6 . Results for $g^{\prime}=\infty$ correspond to the case without the pion in-medium effects.

a slight reduction of the isospin asymmetry to $\delta_{\text {like }} \simeq 0.073$, 0.098 , and 0.135 for the three symmetry energies, given by $x=0,0.5$, and 1 , respectively. We note that with the fugacity of nucleons kept at $z_{N}=1$, the fugacity parameters of about $z_{\pi}=0.0855$ and $z_{\Delta}=0.459$ are needed for the symmetry energy given by $x=1$ to keep the total number of pions as well as that of $\Delta$ resonances the same as in the case without pion in-medium effects and that the required values for the fugacity parameters are similar for the other two symmetry energies considered here.

Results on the $\pi^{-} / \pi^{+}$ratio in $\mathrm{Au}+\mathrm{Au}$ collisions at the beam energy of $0.4 \mathrm{~A} \mathrm{GeV}$ are shown in Fig. 4. With the value $g^{\prime}=1 / 3$ for the Migdal parameter, values for the $\pi^{-} / \pi^{+}$ratio are $2.12,2.30$, and 2.47 for the three symmetry energy parameters $x=0,0.5$, and 1 , respectively, which are slightly smaller than corresponding values for the case without including the pion in-medium effects as shown by those for $g^{\prime}=\infty$ in Fig. 4. The measured $\pi^{-} / \pi^{+}$ratio of about 3 by the FOPI Collaboration, shown in Fig. 4 by the dash-dotted line together with the error bar, which without the inclusion of the pion in-medium effects in the IBUU model favors a nuclear symmetry energy softer than the one given by $x=1$, thus requires an even softer one after including the isospindependent pion in-medium effects. Figure 4 further shows results obtained with larger values of $g^{\prime}=0.4,0.5$, and 0.6 for the Migdal parameter. It is seen that the isospin-dependent pion in-medium effects in these cases are not much different from the case of $g^{\prime}=1 / 3$, indicating that the pion abundance in hot dense asymmetric nuclear matter is affected more by the $s$-wave, which does not depend on $g^{\prime}$, than by the $p$-wave interaction between pion and nucleon.

\section{SUMMARY}

In summary, we studied the dependence of the pion spectral function in asymmetric nuclear matter on the charge of the pion by using results from the chiral perturbation theory for the pion-nucleon $s$-wave interaction and from the $\Delta$-hole model for the pion-nucleon $p$-wave interaction. Because of increasing $\pi^{-}$and decreasing $\pi^{+}$in-medium masses due to the pion-nucleon $s$-wave interaction in neutron-rich matter, the strength of $\pi^{+}$spectral function at low energies is somewhat larger than that of $\pi^{-}$spectral function and the strength around the peak of the $\Delta$ resonance mass distribution decreases while that near the threshold increases with increasing charge of the $\Delta$ resonance. In a thermal model that assumes that nucleons, pions, and $\Delta$ resonances produced in heavy ion collisions are in thermal but not chemical equilibrium, with the latter needed to maintain the final pion to nucleon ratio, the $\pi^{-} / \pi^{+}$ratio is slightly reduced in comparison with the case without pion in-medium effects. Taking into consideration of the isospin-dependent pion in-medium effects in the transport model thus will have some, albeit not very significant, effects on the extraction of the nuclear symmetry energy from measured $\pi^{-} / \pi^{+}$ratio. However, the predicted $\pi^{-} / \pi^{+}$ratio for a given parametrization of the symmetry energy can be quite different in different transport models. For example, it was shown in Ref. [14] that the $\pi^{-} / \pi^{+}$ ratio in hot dense nuclear matter increases with increasing stiffness of the nuclear symmetry energy at high densities if the latter is parameterized using the relativistic mean-field model, opposite to the results from the IBUU model [15], which shows a decreasing $\pi^{-} / \pi^{+}$ratio with increasing stiffness of the nuclear symmetry energy. Also, it was recently shown in an improved isospin-dependent quantum molecular dynamic (ImIQMD) model that the measured $\pi^{-} / \pi^{+}$ratio from the FOPI Collaboration is consistent with a nuclear symmetry energy that is even stiffer than the one corresponding to the symmetry energy parameter $x=0$ in the MDI interaction [31], contradictory to the conclusion from the IBUU model [15] that a symmetry energy softer than $x=1$ is needed to reproduce the measured $\pi^{-} / \pi^{+}$ratio. Further theoretical work is thus needed to understand the relation between the $\pi^{-} / \pi^{+}$ratio and the behavior of the nuclear symmetry energy at high densities in the transport model description of heavy ion collisions.

\section{ACKNOWLEDGMENTS}

This work was supported in part by the US National Science Foundation under Grant No. PHY-0758115 and the Welch Foundation under Grant No. A-1358.
[1] B. A. Li, L. W. Chen, and C. M. Ko, Phys. Rep. 464, 113 (2008).

[2] B. A. Li, C. M. Ko, and W. Bauer, Int. J. Mod. Phys. E 7, 147 (1998).

[3] Isospin Physics in Heavy Ion Collisions at Intermediate Energies, edited by B. A. Li and W. Udo Schröder (Nova Science Publishers, New York, 2001).
[4] V. Baran, M. Colonna, V. Greco, and M. Di Toro, Phys. Rep. 410, 335 (2005).

[5] M. B. Tsang et al., Phys. Rev. Lett. 92, 062701 (2004).

[6] T. X. Liu et al., Phys. Rev. C 76, 034603 (2007).

[7] L. W. Chen, C. M. Ko, and B. A. Li, Phys. Rev. Lett. 94, 032701 (2005). 
[8] B. A. Li and L. W. Chen, Phys. Rev. C 72, 064611 (2005).

[9] D. V. Shetty, S. J. Yennello, and G. A. Souliotis, Phys. Rev. C 75, 034602 (2007).

[10] A. W. Steiner and B. A. Li, Phys. Rev. C 72, 041601(R) (2005).

[11] T. Li et al., Phys. Rev. Lett. 99, 162503 (2007).

[12] B. A. Li, Phys. Rev. Lett. 88, 192701 (2002); Nucl. Phys. A708, 365 (2002).

[13] G. Ferini, T. Gaitanos, M. Colonna, M. Di Toro, and H. H. Wolter, Phys. Rev. Lett. 97, 202301 (2006).

[14] G. Ferini, M. Colonna, T. Gaitanos, and M. Di Toro, Nucl. Phys. A762, 147 (2005).

[15] Z. G. Xiao, B. A. Li, L. W. Chen, G. C. Yong, and M. Zhang, Phys. Rev. Lett. 102, 062502 (2009); M. Zhang, Z. G. Xiao, B. A. Li, L. W. Chen, G. C. Yong, and S. J. Zhu, Phys. Rev. C 80, 034616 (2009).

[16] W. Reisdorf et al. (FOPI Collaboration), Nucl. Phys. A781, 459 (2007).

[17] G. E. Brown and W. Weise, Phys. Rep. 22, 279 (1975).

[18] B. Friedmann, V. R. Pandharipande, and Q. N. Usmani, Nucl. Phys. A372, 483 (1981).

[19] E. Oset, H. Toki, and W. Weise, Phys. Rep. 83, 281 (1982).
[20] L. H. Xia, P. Siemens, and M. Soyeur, Nucl. Phys. A578, 493 (1994).

[21] H. van Hees and R. Rapp, Phys. Lett. B606, 59 (2005).

[22] C. L. Korpa, M. F. M. Lutz, and F. Riek, Phys. Rev. C 80, 024901 (2009).

[23] C. L. Korpa and A. E. L. Dieperink, Phys. Lett. B446, 15 (1999).

[24] L. Xiong, C. M. Ko, and V. Koch, Phys. Rev. C 47, 788 (1993).

[25] C. Fuchs, L. Sehn, E. Lehmann, J. Zipprich, and A. Faessler, Phys. Rev. C 55, 411 (1997).

[26] N. Kaiser and W. Weise, Phys. Lett. B512, 283 (2001).

[27] C. M. Ko, L. H. Xia, and P. J. Siemens, Phys. Lett. B231, 16 (1989).

[28] B. A. Li and C. M. Ko, Phys. Rev. C 52, 2037 (1995); B. A. Li, A. T. Sustich, B. Zhang, and C. M. Ko, Int. J. Mod. Phys. E 10, 267 (2001).

[29] H. van Pee et al. (CB-ELSA Collaboration), Eur. Phys. J. A 31, 61 (2007).

[30] G. F. Bertsch, Nature (London) 283, 280 (1980); A. Bonasera and G. F. Bertsch, Phys. Lett. B195, 521 (1987).

[31] Z. Q. Feng and G. M. Jin, Phys. Lett. B683, 140 (2010). 